\title{
First interferometric detections of Fast Radio Bursts
}

M. Caleb ${ }^{1,2,3,4}$, C. Flynn ${ }^{2,3}$, M. Bailes ${ }^{2,3}$, E. D. Barr ${ }^{2,3,5}$, T. Bateman ${ }^{7}$, S. Bhandari ${ }^{2,3}$, D. Campbell-Wilson ${ }^{7}$, W. Farah $^{2}$, A. J. Green ${ }^{7,3}$, R. W. Hunstead ${ }^{7}$, A. Jameson ${ }^{2,3}$ F. Jankowski ${ }^{2,3}$, E. F. Keane ${ }^{6}$, A. Parthasarathy ${ }^{2,3}$, V. Ravi ${ }^{2,3,8}$, P. A. Rosado ${ }^{2,9}$, W. van Straten ${ }^{2,10}$ and V. Venkatraman Krishnan ${ }^{2,3}$

${ }^{1}$ Research School of Astronomy and Astrophysics, Australian National University, ACT, 2611, Australia

email: manisha.caleb@manchester.ac.uk

${ }^{2}$ Centre for Astrophysics and Supercomputing, Swinburne University of Technology, P.O. Box 218, Hawthorn, VIC 3122, Australia

${ }^{3}$ ARC Centre of Excellence for All-sky Astrophysics (CAASTRO)

${ }^{4}$ Jodrell Bank Centre for Astrophysics, School of Physics and Astronomy, The University of Manchester, Manchester M13 9PL, UK

${ }^{5}$ Max-Planck-Institut für Radioastronomie, Auf dem Hügel 69, D-53121 Bonn, Germany

${ }^{6}$ SKA Organisation, Jodrell Bank Observatory, Cheshire, SK11 9DL, UK

${ }^{7}$ Sydney Institute for Astronomy (SIfA), School of Physics, The University of Sydney, NSW 2006, Australia

${ }^{8}$ Cahill Center for Astronomy and Astrophysics, MC249-17, California Institute of Technology, Pasadena, CA 91125, USA

${ }^{9}$ Monash Centre for Astrophysics, School of Physics and Astronomy, Monash University, VIC 3800, Australia

${ }^{10}$ Institute for Radio Astronomy \& Space Research, Auckland University of Technology, Private Bag 92006, Auckland 1142, New Zealand

\begin{abstract}
The class of radio transients called Fast Radio Bursts (FRBs) encompasses enigmatic single pulses, each unique in its own way, hindering a consensus for their origin. The key to demystifying FRBs lies in discovering many of them in order to identity commonalities - and in real time, in order to find potential counterparts at other wavelengths. The recently upgraded UTMOST in Australia, is undergoing a backend transformation to rise as a fast transient detection machine. The first interferometric detections of FRBs with UTMOST, place their origin beyond the near-field region of the telescope thus ruling out local sources of interference as a possible origin. We have localised these bursts to much better than the ones discovered at the Parkes radio telescope and have plans to upgrade UTMOST to be capable of much better localisation still.
\end{abstract}

Keywords. instrumentation: interferometers, intergalactic medium, surveys

\section{Introduction}

Fast radio bursts (FRBs) are a relatively new class of radio transients comprising of pulses (typically non-repeating) which are short $(\sim \mathrm{ms})$, bright $(\sim$ few Jy) and exhibiting dispersion sweeps characteristic of propagation through a cold ionised diffuse plasma (Lorimer et al. 2007). Most published FRBs with the exception of FRB 121102 (Spitler et al. 2016) have been detected with single dish antennas, with relatively poor angular resolution, and we are unable to indisputably rule out a near-field or atmospheric origin 


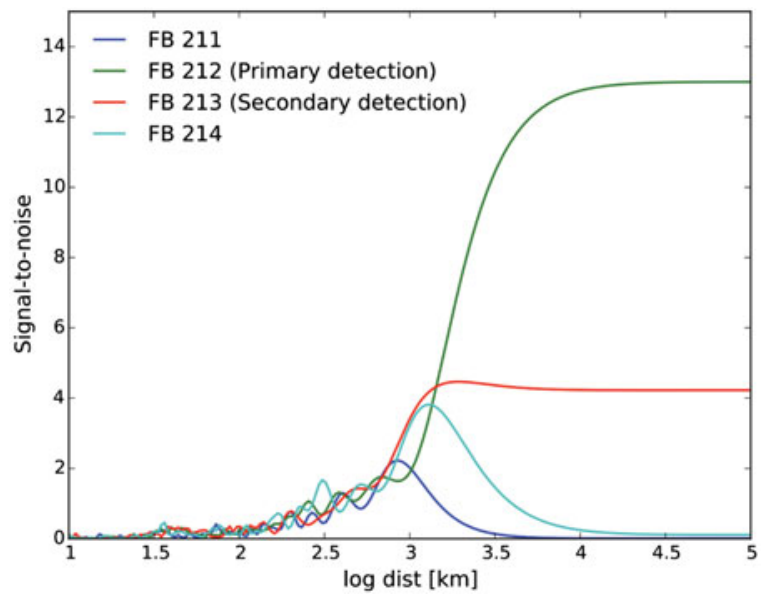

Figure 1. Determination the Fresnel limit of UTMOST through simulations of the detections of FRB 160317 in adjacent fan-beams (FB). The S/Ns of the modelled pulse in FB 212 and FB 213 , along with the non-detections in other FBs, match that of the observations at a distance of $\gtrsim 10^{4} \mathrm{~km}$. Figure taken from Caleb et al. 2017.

for the non-repeating events, until now. The Molonglo Observatory Synthesis Telescope in Australia is undergoing a major upgrade to transform it into an FRB finding machine - the UTMOST. In 2016, UTMOST made the first interferometric detections of 3 FRBs in a 180-day survey of the Southern sky which further strengthened their case for an astronomical origin.

\section{Survey specifications and results}

UTMOST is an E-W array which operates at $843 \mathrm{MHz}$ with a $31.25 \mathrm{MHz}$ bandwidth and single polarization (right circular). The $\sim 9 \mathrm{deg}^{2}$ primary beam of the telescope is tiled with 352 elliptical, coherent, tied-array beams (called 'fan-beams', each 46" wide and overlapping at close to their half power points). The resolution of the fan-beams in the N-S direction is the same as that of the primary beam $(\sim 2.8$ degrees $)$. The adjacent fan-beam detections of FRBs 160317, 160410, 160608 have enabled $3 \sigma$ localization ellipses of $15^{\prime \prime} \times 8.4^{\circ}$ with their orientations hour angle dependent.

Extensive pulsar observations with UTMOST have confirmed that a pulse from a farfield point source is detected in a maximum of 3 adjacent fan-beams at any given time. We have modelled the point of separation between the near-field region and the farfield region of the telescope for FRB 160317 as an example. We simulate the fan-beam responses to a point source as a function of distance, to reproduce the observed $\mathrm{S} / \mathrm{N}$ distribution of FRB 160317 in the detected adjacent fan-beams. In Figure 1 taken from Caleb et al. 2017, we see that a two fan-beam detection with $\mathrm{S} / \mathrm{Ns}$ of $\sim 13$ and $\sim 5$ in the primary and secondary fan-beams of detection respectively, is achieved at $\gtrsim 10^{4} \mathrm{~km}$. Thus detections of FRBs in 1 or 2 fan-beams only allow us to identify them as originating well away from the Earth effectively ruling out local sources of interference as an origin.

\section{References}

Lorimer, D. R., Bailes, M., McLaughlin, M. A., et al., 2007, Science, 318, 777

Spitler, L. G., Scholz, P., Hessels, J. W. T., et al., 2017, Nature, 531, 202

Caleb, M., Flynn, C., Bailes, M., et al., 2017, MNRAS, 468, 3746 